\title{
Functional Genomics and
} Phylogenetic Evidence Suggest Genus-Wide Cobalamin Production by the Globally Distributed Marine Nitrogen Fixer Trichodesmium

\author{
Nathan G. Walworth ${ }^{1 \dagger}$, Michael D. Lee ${ }^{1 \dagger}$, Christopher Suffridge ${ }^{1}$, Pingping $Q u^{1}$, \\ Fei-Xue Fu ${ }^{1}$, Mak A. Saito ${ }^{2}$, Eric A. Webb ${ }^{1}$, Sergio A. Sañudo-Wilhelmy ${ }^{1}$ and \\ David A. Hutchins ${ }^{1 *}$ \\ ${ }^{1}$ Department of Biological Sciences, University of Southern California, Los Angeles, CA, United States, ${ }^{2}$ Marine Chemistry \\ and Geochemistry Department, Woods Hole Oceanographic Institution, Woods Hole, MA, United States
}

OPEN ACCESS

Edited by:

Senjie Lin,

University of Connecticut,

United States

Reviewed by:

Elizabeth Anne Edwards, University of Toronto, Canada

Vicente Mariscal,

Consejo Superior de Investigaciones

Científicas (CSIC), Spain

*Correspondence: David A. Hutchins

dahutch@usc.edu

${ }^{\dagger}$ These authors have contributed equally to this work.

Specialty section: This article was submitted to

Aquatic Microbiology,

a section of the journal

Frontiers in Microbiology

Received: 12 November 2017

Accepted: 26 January 2018

Published: 13 February 2018

Citation:

Walworth NG, Lee MD, Suffridge C, Qu P, Fu F-X, Saito MA, Webb EA,

Sañudo-Wilhelmy SA and

Hutchins DA (2018) Functional Genomics and Phylogenetic Evidence

Suggest Genus-Wide Cobalamin Production by the Globally Distributed Marine Nitrogen Fixer Trichodesmium.

Front. Microbiol. 9:189.

doi: 10.3389/fmicb.2018.00189
Only select prokaryotes can biosynthesize vitamin $\mathrm{B}_{12}$ (i.e., cobalamins), but these organic co-enzymes are required by all microbial life and can be vanishingly scarce across extensive ocean biomes. Although global ocean genome data suggest cyanobacteria to be a major euphotic source of cobalamins, recent studies have highlighted that $>95 \%$ of cyanobacteria can only produce a cobalamin analog, pseudo- $\mathrm{B}_{12}$, due to the absence of the BluB protein that synthesizes the $\alpha$ ligand 5,6-dimethylbenzimidizole (DMB) required to biosynthesize cobalamins. Pseudo- $B_{12}$ is substantially less bioavailable to eukaryotic algae, as only certain taxa can intracellularly remodel it to one of the cobalamins. Here we present phylogenetic, metagenomic, transcriptomic, proteomic, and chemical analyses providing multiple lines of evidence that the nitrogen-fixing cyanobacterium Trichodesmium transcribes and translates the biosynthetic, cobalamin-requiring BluB enzyme. Phylogenetic evidence suggests that the Trichodesmium DMB biosynthesis gene, bluB, is of ancient origin, which could have aided in its ecological differentiation from other nitrogen-fixing cyanobacteria. Additionally, orthologue analyses reveal two genes encoding iron-dependent $\mathrm{B}_{12}$ biosynthetic enzymes (cbiX and isiB), suggesting that iron availability may be linked not only to new nitrogen supplies from nitrogen fixation, but also to $\mathrm{B}_{12}$ inputs by Trichodesmium. These analyses suggest that Trichodesmium contains the genus-wide genomic potential for a previously unrecognized role as a source of cobalamins, which may prove to considerably impact marine biogeochemical cycles.

Keywords: cobalamin, trichodesmium, Vitamin $\mathrm{B}_{12}$, iron limitation, Cyanobacteria, BluB gene, nitrogen fixation

\section{INTRODUCTION}

Marine cyanobacteria and eukaryotic algae are estimated to be responsible for up to $50 \%$ of global carbon fixation, and can be limited by both macronutrients (e.g., nitrogen and phosphorus) and micronutrients (e.g., iron) (Field et al., 1998; Arrigo, 2005; Hutchins et al., 2009; Hutchins and Boyd, 2016). Organic coenzymes known as B-vitamins have also been implicated to limit primary production and influence microbial community structure (Panzeca et al., 2006; SañudoWilhelmy et al., 2014; Suffridge et al., 2017). B-vitamins are soluble, non-protein molecules that 
bind to enzymes to increase reaction rates and are required for essential cellular processes such as DNA repair, redox reactions, photosynthesis, and carbon fixation (Monteverde et al., 2016). Most eukaryotic algae (Droop, 2007) and many heterotrophic bacteria (Giovannoni et al., 2005) have obligate B-vitamin requirements (e.g., auxotrophy) for processes like reductive dehalogenation and fatty acid biosynthesis, with growth requiring assimilation via exogenous sources including breakdown of vitamin-containing cells and/or interactions with vitamin-producing bacteria and archaea (Stadtman et al., 1960; Mohn and Tiedje, 1992; Bertrand et al., 2015; Heal et al., 2016). Microbes that do not have an absolute requirement for $B_{12}$ employ $B_{12}$-independent versions of certain enzymes such as the $\mathrm{B}_{12}$-independent methionine synthase (MetE) and the $\mathrm{B}_{12}$ independent radical SAM DNA methyltransferases (Heal et al., 2016).

Some chemical form of vitamin $\mathrm{B}_{12}$ is required by all microbial life for a range of functions, including methionine biosynthesis, ribonucleotide reduction, photoregulation, and various onecarbon metabolisms (Sañudo-Wilhelmy et al., 2014; Fang et al., 2017). Vitamin $B_{12}$ is a general term referring to cobaltcontaining corrinoids (i.e., molecules containing a corrin ring) that contain upper $(\beta)$ and lower $(\alpha)$ axial ligands to the cobalt ion that can vary depending on functionality (Eschenmoser and Wintner, 1977; Warren et al., 2002; Helliwell et al., 2016). A primary type of $\mathrm{B}_{12}$, cobalamin $(\mathrm{CBL})$, is a complex coenzyme with an $\alpha$ ligand of 5,6-dimethylbenzimidizole (DMB), and a $\beta$ ligand of either an adenosyl-, methyl-, cyanide-, or hydroxylgroup (Ado-, Me-, CN-, or OH-; Heal et al., 2016; Figure 1). For example, methylcobalamin (Me-CBL) has a methyl group as its $\beta$ ligand and is involved in methylation reactions, whereas adenosylcobalamin (Ado-CBL, coenzyme $\mathrm{B}_{12}$ ) has an adenosyl group (5-deoxyadenosine) and is involved in radical-based rearrangements and reductions (Banerjee and Ragsdale, 2003).

Many marine microbes have an absolute requirement for $\mathrm{B}_{12}$ (Bertrand et al., 2013), and locally produced cobalamin is likely the predominant source for microbial assimilation due to its short (hours to days) residence time in the surface ocean (Carlucci et al., 1969; Bertrand et al., 2015). Those microbes that can grow without cobalamins must do so at a metabolic cost, using an alternative methionine synthase (MetE) that is relatively less-efficient than the $\mathrm{B}_{12}$-dependent $\mathrm{MetH}$ enzyme (Helliwell et al., 2016). These biochemical constraints and environmental interactions highlight $\mathrm{B}_{12}$ as a potential driver of ecological niche partitioning in marine environments. Thaumarchaeota (Doxey et al., 2015; Santoro et al., 2015) and both heterotrophic bacteria (Croft et al., 2005) and cyanobacteria (Bonnet et al., 2010) are hypothesized to be major cobalamin producers in the ocean, thereby potentially modulating overall primary productivity. However, recent studies have indicated that most cyanobacteria produce a cobalamin analog, pseudo$\mathrm{B}_{12}$ (Taga and Walker, 2008), where adenine substitutes for DMB as the $\alpha$ ligand (Heal et al., 2016; Helliwell et al., 2016) (Figure 1). The compound DMB is synthesized aerobically by the BluB enzyme, or anaerobically via enzymes of the $b z a$ operon (Hazra et al., 2015; Mehta et al., 2015). A recent assessment found that only one cyanobacterial genome out of 255 contained an annotated bluB gene (Heal et al., 2016), and a targeted search of 118 cyanobacterial genomes found it in only five species, while none contain the bza operon (Helliwell et al., 2016). Indeed, numerous BluB-lacking strains of the globally distributed Synechococcus and Prochlorococcus genera solely biosynthesize pseudo-B 12 (Heal et al., 2016; Helliwell et al., 2016). Additionally, pseudo- $B_{12}$ is used less efficiently than cobalamin for several $\mathrm{B}_{12}$-dependent algae, and only certain algae can intracellularly remodel (i.e., salvage) pseudo- $B_{12}$ to cobalamin in the presence of exogenous DMB (Helliwell et al., 2016). Cultured algae whose growth could be rescued with supplemented pseudo$\mathrm{B}_{12}+\mathrm{DMB}$ could not grow in natural seawater supplemented with pseudo- $\mathrm{B}_{12}$ alone, thereby indicating $\mathrm{DMB}$ concentrations to be insufficient in certain habitats (Helliwell et al., 2016). In contrast, bluB-containing bacteria and archaea synthesize only cobalamin (Heal et al., 2016). Although BluB synthesizes $\mathrm{DMB}$, the enzyme CobT has been found to be necessary for its activation across most microbial phyla studied to date (Croft et al., 2006; Escalante-Semerena, 2007; Yan et al., 2018). The bluBcontaining Thaumarchaeota however solely produce cobalamin despite lacking cobT, demonstrating that DMB activation may occur through a similar enzyme with high sequence divergence or through a different, yet unknown, genetic mechanism (Heal et al., 2016). Trichodesmium may also employ a different activation mechanism than the previously identified CobT, as described below.

Heal et al. (2016) detected both particulate pseudo- $\mathrm{B}_{12}$ and cobalamin in the North Pacific surface ocean at similar concentrations ( 0.01 to $0.1 \mathrm{pM} \mathrm{L}^{-1}$ ), yet solely cobalamin beneath the photic zone. This co-occurrence at the surface suggests that bluB-lacking cyanobacteria synthesize pseudo$\mathrm{B}_{12}$ in the presence of cobalamin, and that cobalamin in the surface ocean could be a result of either de novo synthesis by heterotrophic microbes, or remodeled pseudo- $\mathrm{B}_{12}$ originally from cyanobacteria. Hence, cyanobacteria may gain a competitive advantage by producing and solely requiring pseudo$\mathrm{B}_{12}$ for growth, as this avoids them directly supplying other cobalamin-requiring photoautotrophs.

In contrast, marine nitrogen-fixing cyanobacteria (diazotrophs) directly supply both fixed carbon and nitrogen, thereby impacting marine primary productivity and biogeochemical cycling (Falkowski et al., 1998; Sohm et al., 2011). The globally distributed colony-forming diazotroph, Trichodesmium, is among the most important global contributors of bioavailable nitrogen in the oligotrophic oceans with some estimates suggesting it to make up as much as half of total $\mathrm{N}_{2}$ fixation in the vast subtropical gyre regions (Zehr, 2011; Hutchins et al., 2015). Trichodesmium can form extensive, recurring blooms in the Arabian and Red seas and Atlantic and Pacific oceans (Walworth et al., 2015). It serves as a millimetersized substrate for prokaryotic and eukaryotic microbial consortia (Bergman et al., 2013; Lee et al., 2017a) and also provides organic metabolites via excretion (Capone et al., 1994). Another common albeit less prevalent marine diazotroph, the unicellular genus Crocosphaera, excretes B-vitamins in culture at rates that exceed those of non-diazotrophic cyanobacteria (Bonnet et al., 2010), and recent studies have shown excretion of 
A

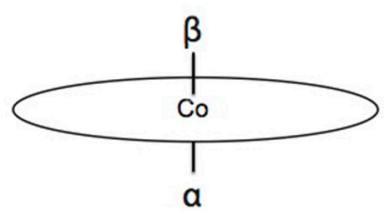

B

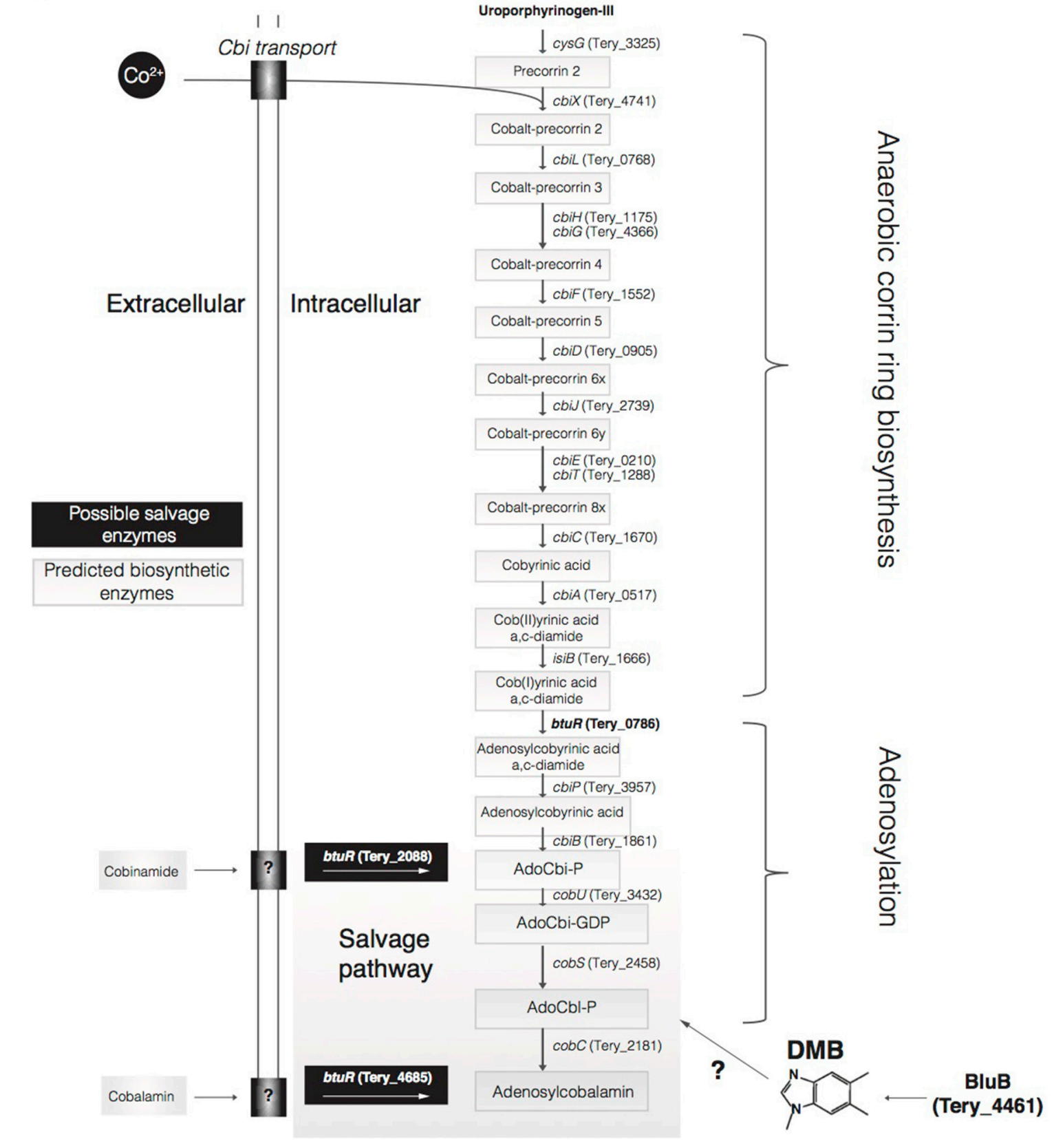

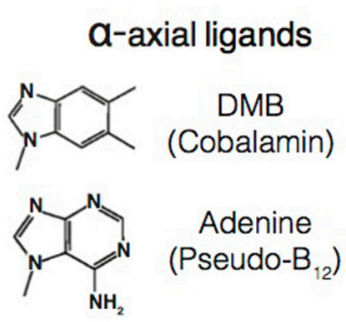

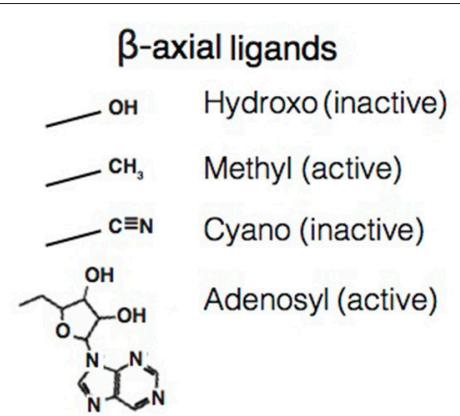

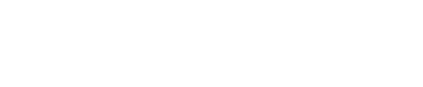
(1)

FIGURE 1 | Diagram of different forms of cobalamin, Psuedo- $B_{12}$ and proposed cobalamin biosynthetic and salvage pathways. (A) The ellipse represents the cobalt-containing corrin ring, and listed are the various $\alpha$-axial and $\beta$-axial ligands. (B) Displayed is the fully predicted pathway for $\mathrm{B}_{12}$ biosynthesis including potential salvage pathways. 
B-vitamin precursors in marine heterotrophic bacteria known to associate with phytoplankton blooms (Wienhausen et al., 2017). Despite intensive study on the global distribution and ecophysiology of Trichodesmium, knowledge about its capacity to biosynthesize and salvage critical cobalamin-type coenzymes is lacking.

Recently, we conducted a 7-year experimental evolution study adapting Trichodesmium erythraeum IMS101 (IMS101) to high $\mathrm{CO}_{2}$ under multiple limiting nutrient regimes. We generated a series of functional genomic datasets to explore both its molecular evolution (Hutchins et al., 2015; Walworth et al., 2016) and its ecological relationship with its epibiotic consortia (Lee et al., 2017a). While not originally intended to examine B-vitamin metabolism, we conducted a metaanalysis of our functional genomic datasets combined with other Trichodesmium metagenomic (Walworth et al., 2015), and phylogenetic data, which revealed strong evidence that the Trichodesmium genus can biosynthesize and salvage cobalamin in situ. Additionally, we substantiate molecular results by measuring intracellular cobalamin concentrations from our remaining long-term Trichodesmium samples using liquid chromatography coupled to mass spectrometry (LCMS) (Suffridge et al., 2017), thereby providing evidence of metabolized cobalamin. Corrin ring biosynthesis and adenosylation is conserved among cyanobacteria (Heal et al., 2016) indicating their presence within the phylum when it originally diverged. We conducted further phylogenetic analyses to explore the potential alternative origins of the $b l u B$ gene necessary for DMB biosynthesis, which may help elucidate $\mathrm{B}_{12}$ niche partitioning of Trichodesmium from co-occurring, sympatric cyanobacterial taxa including other diazotrophs and the cosmopolitan non- $\mathrm{N}_{2}$-fixing genera, Prochlorococcus and Synechococcus. We also describe $\mathrm{B}_{12}$-associated transcripts deriving from the Trichodesmium bacterial epibiotic community to examine a possible role of intra-colony cobalamin cycling. This previously unreported (Capone et al., 1997; Sohm et al., 2011; Bergman et al., 2013; Walworth et al., 2015) potential source of cobalamin deriving from either Trichodesmium, its epibionts, or both provide evidence for yet another critical role that Trichodesmium may serve by supplying a potentially limiting organic micronutrient to the surrounding microbial community. Further, comparative phylogenetic analyses highlight divergent sequence domains of cyanobacterial $\mathrm{B}_{12}$ biosynthetic and salvage pathways relative to other sequenced prokaryotes. Finally, we use comparative orthologue analyses to highlight the possible association of two $\mathrm{B}_{12}$ biosynthetic genes with environmental iron supply, suggesting simultaneous influence of both Trichodesmium nitrogen fixation and $\mathrm{B}_{12}$ production by this limiting trace element.

\section{MATERIALS AND METHODS}

\section{Sequence Analyses}

Gene sequences for the cultured $T$. erythraeum isolates IMS101 and 2175 were downloaded from the Integrated Microbial Genomes (IMG) website (https://img.jgi.doe.gov/), and Trichodesmium environmental metagenomic sequence data was used from Walworth et al. 2015 (Walworth et al., 2015). Sequences were searched against the RefSeq protein database (Tatusova et al., 2015) using the BLASTP algorithm (Altschul et al., 1990), and all high-scoring pairs were retained if the aligned portion spanned $>70 \%$ of the original query length with an evalue $<10^{-5}$. Duplicate sequences were removed with USEARCH (Edgar, 2010). All sequences were aligned with MUSCLE v3.8.31 with default settings (Edgar, 2004), and spurious sequences and poorly aligned regions were removed with trimAl 1.2rev59 (Capella-Gutiérrez et al., 2009). RAxML (Stamatakis, 2015) was used for all maximum likelihood phylogenetic analyses with the following settings: -f a -p 12345 -m PROTCATLG -N 100 -x 12345.

\section{Culturing and Molecular Analyses}

Culturing and sampling for RNA and protein of T. erythraeum IMS101 was done as previously described (Walworth et al., 2015). Briefly, semi-continuous cultures growing in replete Aquil media ( $0.37 \mathrm{nM}$ of added $\mathrm{B}_{12}$ as cyanocobalamin) without added fixed nitrogen and a 12:12 light:dark cycle (light intensity of $120 \mu \mathrm{mol}$ photons per meter squared per second) at $26^{\circ} \mathrm{C}$ were filtered in biological duplicate at midday onto $5 \mathrm{um}$ polycarbonate filters (Whatman), immediately flash frozen, and stored in liquid nitrogen. In an additional set of experiments, triplicate cultures were grown for $\sim 20$ generations using the same growth medium, but without added cyanocobalamin, to determine if IMS101 can grow in culture without a supplementary source of $\mathrm{B}_{12}$.

RNA was extracted using the Ambion MirVana miRNA Isolation Kit (Thermo Fisher Scientific) in an RNAse free environment as per the manufacturer's instructions, followed by Ambion's Turbo DNA-free kit to degrade trace amounts of DNA. RNA was then submitted to the UC San Diego Institute for Genomic Medicine (IGM) core for library preparation and sequencing (http://igm.ucsd.edu/genomics/ services.shtml). Briefly, rRNA removal and library construction was done with the TruSeq Stranded RNA Library Prep kit (Illumina), and multiplexed libraries were sequenced using the Illumina Hi-Seq yielding single-end, 50-base pair reads. Raw fastq files were quality trimmed and filtered with Trimmomatic version 0.35 (Bolger et al., 2014) with the following settings: SE -threads 35 -phred33 LEADING:3 TRAILING:3 SLIDINGWINDOW:4:15 MINLEN:35. Trimmed fastq files were then mapped onto IMS101 IMG-called genes (https://img.jgi. doe.gov/) using Bowtie2 v2.2.5 with default settings (Langmead and Salzberg, 2012), and the resulting count matrix was subjected to TMM normalization using edgeR (Robinson et al., 2010). Please see Supplementary Information for consortia read processing and annotation. Protein spectral counts were downloaded directly from a previously published proteome study using these same IMS101 cell lines (Walworth et al., 2016).

\section{Particulate B-Vitamin Analyses}

Cobalamins were extracted from biological duplicate frozen filters from long-term IMS101 cultures grown in seawater medium containing containing $0.37 \mathrm{nM}$ cyanocobalamin (Walworth et al., 2016), as previously described (Suffridge 
et al., 2017). See Supplementary Information for a brief method description.

\section{RESULTS AND DISCUSSION}

\section{Evidence for Cobalamin Biosynthesis in Trichodesmium}

The bluB gene required for aerobic DMB synthesis for cobalamin production has only been detected in five cyanobacterial species (Helliwell et al., 2016), none of which are abundant or quantitatively important in marine ecosystems. Phylogenetic analysis reveals that Trichodesmium bluB homologs from two $T$. erythraeum strains isolated 10 years apart (IMS101 and 21-75) (Walworth et al., 2015) and an environmental Trichodesmium metagenomic sample form their own clade among heterotrophic bacteria (Figure 2). While it is difficult to speculate as to whether the other cyanobacteria possessing $b l u B$ homologs acquired them independently or if other cyanobacteria have selectively lost their copies, this analysis suggests the possibility that bluB has been frequently horizontally transferred and retained between many members of the Proteobacteria and Bacteroidetes phylum (Figure 2).

RNA and protein extracted midday from long-term IMS101 cultures growing semi-continuously (Walworth et al., 2016) demonstrate $b l u B$ to be both actively transcribed and translated into protein (Table S1). The detection of the BluB protein provides strong evidence for active DMB biosynthesis because although genes can be constitutively transcribed at basal levels, bioenergetic investment dramatically increases at the translational level (Lynch and Marinov, 2015). Additionally, since half-lives of bacterial mRNA transcripts are typically short-lived relative to proteins (Rauhut and Klug, 1999), detection of both bluB transcripts and BluB proteins lend further evidence of persistent activity. Furthermore, all $B_{12}$ biosynthetic genes were actively expressed, of which numerous corresponding protein products were also detected (Table S1). Interestingly, the $\mathrm{B}_{12}$-dependent $(\mathrm{met} H)$ and $\mathrm{B}_{12}$-independent (metE) methionine synthases were also both transcribed and translated in the presence of exogenously supplied cobalamin with metE transcripts and protein levels being $\sim 10 \mathrm{X}\left(p<10^{-4}\right)$ and $\sim 5 \mathrm{X}(p<0.05)$ higher, respectively, than those of $m e t H$ (Figure 3A). Prior analysis of bacterial MetE suggests reduced enzyme efficiency relative to MetH (Bertrand et al., 2013; Helliwell et al., 2016), which may explain the increased abundances of MetE transcripts and protein relative to those of MetH. Nonetheless, it is noteworthy that transcripts and proteins of both forms were detected in the presence of cobalamin, suggesting Trichodesmium to persistently utilize both versions of the enzyme irrespective of environmental cobalamin supply.

Additionally, we applied a recently developed method that measures particulate $B_{12}$ concentrations directly from cell lysates to the remaining samples from the long-term Trichodesmium experiment (Walworth et al., 2016), which detected intracellular cobalamins in IMS101 trichomes at a concentration of $\sim 58 \mathrm{pM}$ (Figure S1) (Suffridge et al., 2017). Hence, these measurements suggest the ability of IMS101 to metabolize cobalamins either from salvage or biosynthesis pathways. To assess if physiology is impacted without exogenously supplied $B_{12}$, we performed additional physiology experiments examining growth and nitrogen fixation rates of IMS101 with and without added cobalamin (Methods) and found no difference in either condition (Figure 3B; $p>0.05$ ). Hence, Trichodesmium does not require added $B_{12}$ in culture medium, which further supports an ability to biosynthesize and/or salvage $B_{12}$.

Notably, both Trichodesmium trichomes and colonies (i.e., aggregated trichomes) harbor physically attached microbial consortia comprised of hetero- and phototrophs, including other cyanobacteria, both in culture (Lee et al., 2017a,b) and in situ (Hewson et al., 2009; Rouco et al., 2016). To our knowledge no axenic Trichodesmium cultures currently exist. Because it is therefore impossible to absolutely differentiate potential sources of cobalamin deriving from either IMS101 itself or its microbial epibionts, the active production of both the BluB protein and its transcripts in Trichodesmium cells provides the clearest evidence for cobalamin production in IMS101. Additionally, although the biomass and proportional expression of other cyanobacteria is exceedingly low relative to that of Trichodesmium in colonies (Lee et al., 2017a; $75-80 \%$ of RNA sequencing reads mapped to the IMS101 genome with the remaining mapping to the rest of the consortia), it would also be prohibitively difficult to completely disentangle sources of pseudo- $\mathrm{B}_{12}$ in the particulate fraction if other cyanobacteria were indeed attached to IMS101.

As in naturally occurring colonies isolated in situ (Hewson et al., 2009), our IMS101 cultures contain trace amounts of Synechococcus (Lee et al., 2017a), thereby obstructing the ability to specifically detect pseudo- $B_{12}$ from Trichodesmium. We also detected transcripts of $\mathrm{B}_{12}$ biosynthesis/salvage genes deriving from both Synechococcus and heterotrophic genera in the Trichodesmium epibiotic community (Table S1). Interestingly, only the btuR gene was detected in heterotrophic bacterial transcripts, which can be involved in either biosynthesis or salvage pathways (see below) while both $b t u R$ and the $\mathrm{B}_{12}$ biosynthetic gene, $c b i B$, was detected in cyanobacterial transcripts suggesting production of pseudo- $B_{12}$ by Synechococcus. However, since no other $\mathrm{B}_{12}$ biosynthesis genes were detected in heterotrophic transcripts other than $b t u R$, this opens the door for further investigation pertaining to the sources and sinks of cobalamins between Trichodesmium and its heterotrophic epibionts. A recent genome analysis of IMS101 heterotrophic epibionts revealed that an associated Alteromonas macleodii genome from this isolate is indeed a $\mathrm{B}_{12}$ auxotroph (Lee et al., 2017a). Moreover, the detection of consortia btuR genes may have implications for the use of organically complexed cobalt, which dominates cobalt speciation in most euphotic zone environments (Saito et al., 2005). Additional studies with Trichodesmium colonies devoid of other cyanobacteria coupled to direct measurements of both cobalamin and pseudo- $\mathrm{B}_{12}$ are necessary to determine whether these two forms are simultaneously produced by IMS101 in the particulate fraction.

Further evidence for the ability of Trichodesmium to produce cobalamins comes from the widespread conservation of the bluB gene in Trichodesmium genomes sampled directly in situ 


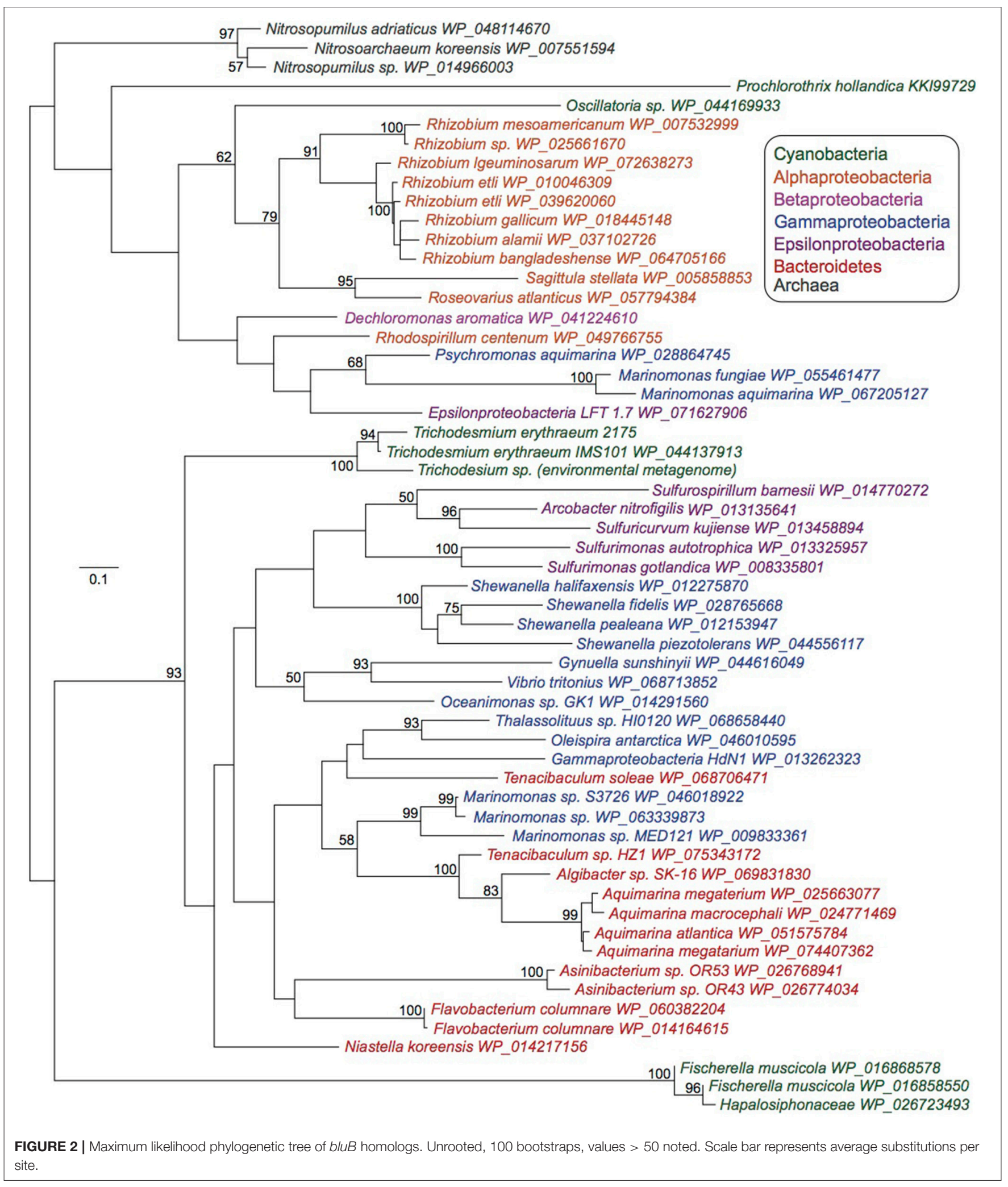

and in the genome of T. erythraeum strain 2175 isolated 10 years after IMS101 (Figure 2). Upon searching a Trichodesmium metagenome sequenced from hand-picked natural colonies
(Walworth et al., 2015), the bluB gene was detected via BLAST(Altschul et al., 1990) with $90 \%$ similarity and evalue $\leq 10^{-123}$. Accordingly, BLAST searches with this metagenomic 

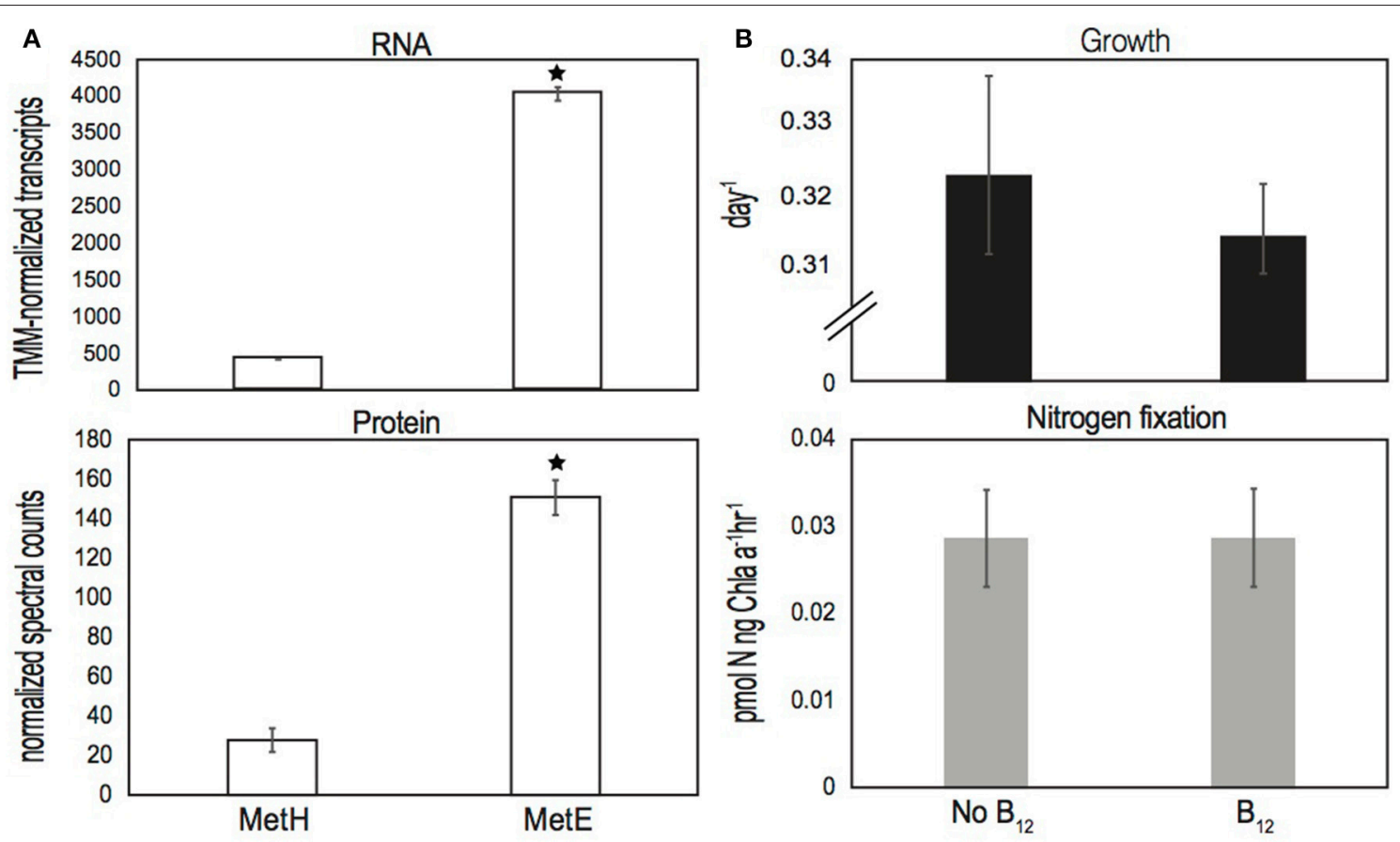

FIGURE 3 | (A) Normalized RNA transcript and protein spectral counts of MetH and MetE from cultures grown in the presence of cyanocobalamin, respectively, and (B) growth and $\mathrm{N}_{2}$ fixation data of IMS101 after 20 generations of growth either with and without added cyanocobalamin. Error bars represent standard deviations of three biological replicates per treatment, and an asterisk $\left(^{*}\right)$ denotes significant differences $(p<0.05)$. Units of $\mathrm{N}_{2}$ fixation are in picomole nitrogen per nanogram of chlorophyll-a per hour.

BluB homolog against the NCBI non-redundant protein database returned IMS101 as its best hit followed by other BluB homologs in other bacterial phyla. These analyses demonstrate retention of the $b l u B$ gene in natural populations, perhaps suggesting that its maintenance has been selected for over time.

Taken together, these data suggest that Trichodesmium genus produces the DMB-producing enzyme BluB in culture and maintains the genetic capability in situ, thereby highlighting yet another possible critical keystone service provided by Trichodesmium to microbial communities. Further study investigating both cobalamin and pseudo- $\mathrm{B}_{12}$ cycling in Trichodesmium colonies is needed to better characterize this underappreciated source of cobalamin in the global oceans.

\section{Phylogenetics of btuR-B 12 Biosynthesis and Salvage Pathways}

To date, biosynthesis of the corrin ring of the vitamin has been found to occur via either an oxygen-dependent (aerobic) or oxygen-independent (anaerobic) pathway (Rodionov, 2003). The production and use of (pseudo)cobalamin is predicted to predate oxygenic photosynthesis, and its genetic capacity is found in virtually all cyanobacterial genomes that have been analyzed (Heal et al., 2016). This suggests the pathway was likely present in the earliest members of the lineage and has been maintained through purifying selection over time. Based on phylogenetic analyses of several corrin ring biosynthesis genes of T. erythraeum strains IMS101 and 2175 (Figures S2-S4),
Trichodesmium indeed retains the $\mathrm{O}_{2}$-independent pathway with all genes clustering deeply within a monophyletic cyanobacterial clade. This also holds true for genes involved in adenosylation following corrin biosynthesis to make adenosylcobalamin (Ado$\mathrm{CBL}$ ), an active form of cobalamin (Figures S5-S7).

Ado-CBL can either be salvaged via the adenosylation of exogenous corrinoids [e.g., cobinamide (Cbi)] or synthesized de novo. Both of these avenues require ATP:corrinoid adenosyltransferase encoded by the btuR gene (Rodionov, 2003). Transport of exogenous corrinoids is facilitated by the highly specific BtuBFCD system in many gram-negative bacteria (Escalante-Semerena, 2007) followed by further processing by the BtuR/CobA, CobU, CobS, and CobC enzymes (Figure 1; Rodionov, 2003; Escalante-Semerena, 2007). BtuR adenosylates a cobalamin precursor in the biosynthetic pathway, or other intermediates via the salvage pathway, to yield adenosylcobinamide (AdoCbi) (Rodionov, 2003; Fang et al., 2017; Figure 1). In the salvage pathway, catalysis by the bifunctional CobU enzyme (Tery_3432) yields AdenosylGDP-cobinamide (Figure 1), which is a true intermediate of the biosynthetic pathway (Woodson and Escalante-Semerena, 2003). Although Trichodesmium does not harbor the BtuBFCD transport system, btuR, cobU, cobS, and $\operatorname{cobC}$ homologs are present in the genome suggesting Trichodesmium may have the capacity to salvage exogenous cobalamin with transport potentially mediated by an alternative mechanism. Furthermore, transcription of all salvage genes was detected in addition 
to protein products of $c o b C$ and all three copies of btuR in Trichodesmium (Table S1), demonstrating salvage-specific enzyme activity in the presence of exogenous cobalamin.

Trichodesmium and other cyanobacteria contain three separate copies of genes retaining $b t u R$ domains, which are broadly distributed throughout the IMS101 genome. Two of these (Tery_0786, Tery_2088) are predicted to be 190 and 178 amino acids long, respectively, while the third (Tery_4685) harbors duplicated btuR catalytic domains and is roughly twice the size. The well-characterized $b t u R / c o b A$ homologs of Escherichia coli and Salmonella typhimurium fall within the Tery_0786 cluster, suggesting this gene in Trichodesmium may also be involved in adenosylating cobinamide. While possessing two single-domain proteins is common among organisms beyond the Cyanobacteria, Tery_4685 and its homologs (all with duplicated domains) are almost exclusively unique to the phylum. The only two known exceptions to this include a chromatophore gene within the Eukaryote Paulina chromatophora, believed to have recently undergone an independent endosymbiotic event taking in a cyanobacterium (Nowack et al., 2008), and a cyanophage derived from Prochlorococcus that, of only 131 predicted proteins, possesses a dual-btuR-domain homolog (Sullivan et al., 2009). Aside from these extraordinary exceptions, this highly conserved, monophyletic distribution suggests that this intragene duplication event may have occurred after Cyanobacteria diverged, but very early in the phylum's lineage.

The concurrent maintenance of all three of these phylogenetically distinct $b t u R$-domain containing genes within the Cyanobacteria suggests they may carry out different rolespossibly with unique specificities for adenosylating varying compounds (e.g., cobinamide/cob(I)alamin/cob(I)yrinate diamide) - and stands as evidence of the successful duplication history this specific domain has experienced. To investigate this, each individual domain, as determined by its alignment to the encompassing protein family (pfam02572), was searched separately against the Refseq protein database (BLASTp) and phylogenetic analysis was performed. This revealed distinct, monophyletic clades for each of the co-occurring domains of Tery_4685 which, along with their conserved spatial relationship, provides further evidence they may have been under divergent yet connected evolutionary pressures (Figure 4).

Furthermore, we detected two distinct clades of homologs to Tery_2088 and Tery_0786 with the former being comprised entirely of Cyanobacteria, and the latter, while possessing a monophyletic clade of Cyanobacteria, being overall taxonomically diverse (Figure 4). While further investigation is required for any mechanistic insight into their roles, considering the conservation of Tery_2088 and Tery_4685 (sequencewise and distribution-wise), they may be essential to the cyanobacterial eco-physiology.

\section{Association of $B_{12}$ Biosynthesis with Iron Bioavailability}

De novo synthesis of vitamin $B_{12}$ requires cobalt, whose total dissolved concentrations in the open ocean are in the picomolar range (5-105 $\mathrm{pM} \mathrm{L}^{-1}$ ) (Knauer et al., 1982) These levels are even lower than those considering limiting for other bioactive trace elements like iron ( $200 \mathrm{pM} \mathrm{L}^{-1}$ ) (Hutchins and Boyd, 2016). Consistent with those low levels of cobalt in the ocean, Panzeca et al. (2008) observed the enhancement of $B_{12}$ production in cobalt amendment experiments, providing strong evidence that the synthesis of this vitamin could be limited by the availability of this trace element (Panzeca et al., 2008). However, the bacterial multifunctional enzyme $\mathrm{CbiX}^{\mathrm{L}}$, which catalyzes the insertion of cobalt in $\mathrm{B}_{12}$ biosynthesis, contains an iron-sulfur cluster (4Fe-4S), thereby implicating $\mathrm{B}_{12}$ biosynthesis to be associated with iron availability (Leech et al., 2003). Archaea have been demonstrated to contain a comparatively smaller $\mathrm{CbiX}\left(\mathrm{CbiX}^{\mathrm{S}}\right)$ enzyme ranging between 120 and 145 amino acids (aa), whereas the bacterial $\mathrm{CbiX}^{\mathrm{L}}$ typically contains $>300$ aa's (Leech et al., 2003).

Trichodesmium, as well as other Cyanobacteria, contain two phylogenetically distinct copies (Figure S2) of the cbiX gene (Tery_4741 and Tery_4427)_of which the former contains an iron-sulfur cluster MXCXXC metal-binding motif (4Fe-4S) (Leech et al., 2003), while the latter does not (e.g., presumably not iron-sulfur containing; Figure S8). Both copies were indeed found to be transcribed indicating simultaneous expression, although their protein products were not detected (Table S1). Interestingly, only the longer Tery_4741 (347 aa) is homologous to annotated Archaea $\mathrm{CbiX}^{\mathrm{S}}$ proteins harboring conserved MXCXXC motifs, while no homology was detected with the shorter Tery_4427 (293 aa) when searched against all Archaea via BLASTP. Both Tery_4741 and Tery_4427 contain CbiX and Sirohydrochlorin ferrochelatase (SirB) domains, yet Tery_4741 solely retains both the $\mathrm{MXCXXC}$ motif (Figure S8) and a C-terminal histidine-rich region typically diagnostic of $\mathrm{CbiX}^{\mathrm{L}}$ (Brindley et al., 2003; Leech et al., 2003). SirB, involved in siroheme biosynthesis, shares homology with CbiX but lacks the C-terminal histidine-rich region resulting in a substantially shorter peptide (Leech et al., 2003). Interestingly, although Tery_4427 could have SirB functionality since it lacks the Cterminal histidine-rich region yet is homologous to Tery_4741 (Figure S8), homology was only detected between Tery_4741 and the Bacillus megatarium SirB homolog. Phylogenetic analysis places the Tery_4741 gene in a taxonomically mixed clade comprised of mostly Cyanobacteria and other gram-positive bacteria, while Tery_4427 resides within a clade comprised of nearly all Cyanobacteria except for several gram-positive ones (Figure S2). Additionally, many taxa contain multiple cbiX gene copies within a single genome. The presence of two phylogenetically distinct copies (Figure S2) may have implications for ecological strategy in microbial cobalamin production where reallocation of iron is enabled by functional substitution of the iron-sulfur-containing CbiX protein. Similar iron-sparing strategies such as replacement of the iron-sulfur protein electron carrier ferredoxin with non-iron-containing flavodoxin have been demonstrated in cyanobacteria and other photoautotrophs (Chappell and Webb, 2010; Chappell et al., 2012; Morrissey and Bowler, 2012). Since many different ironcontaining proteins are involved in broad metabolic pathways throughout the cell such as photosynthesis, nitrogen fixation, 


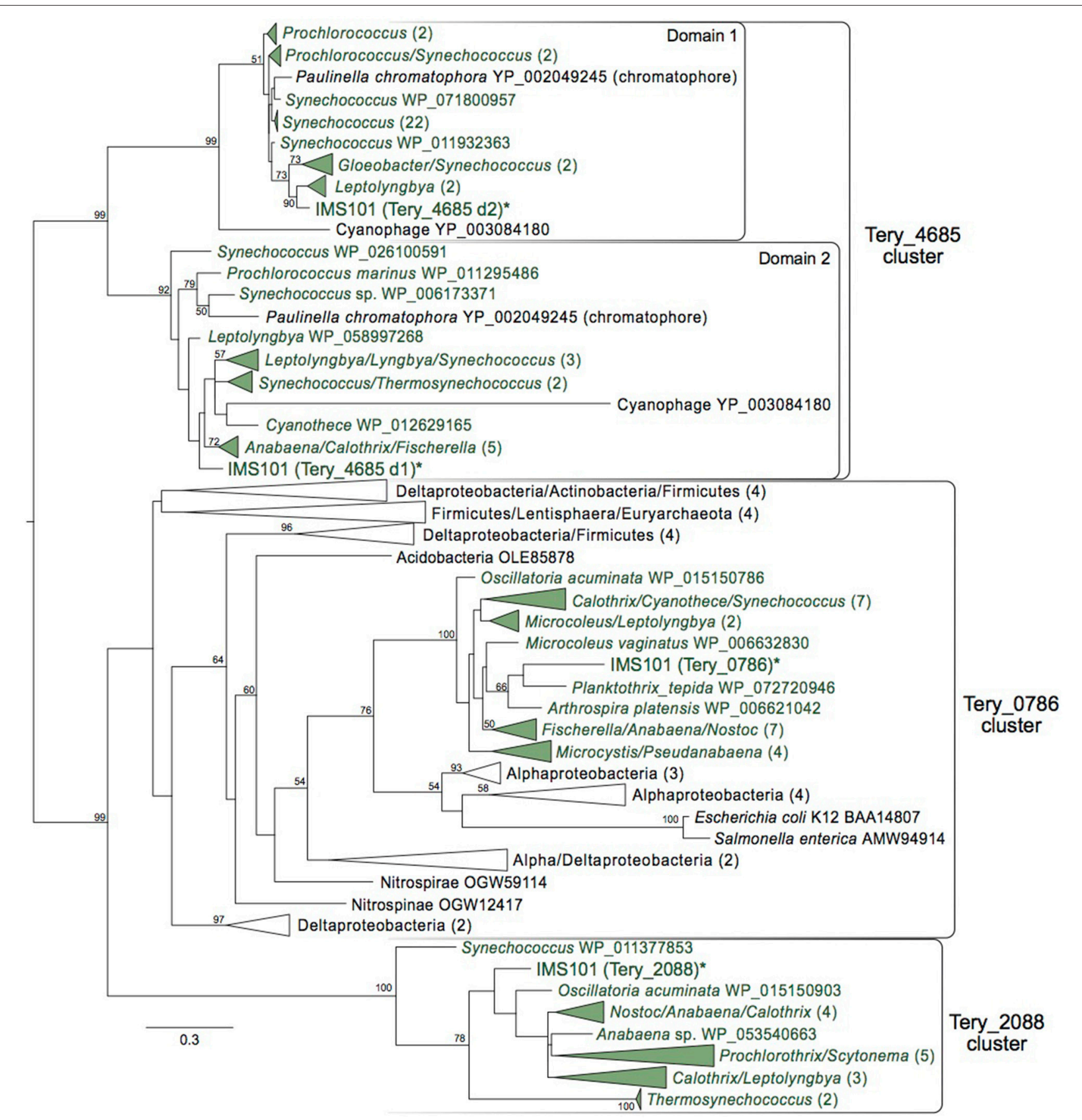

FIGURE 4 | All three copies of btuR in IMS101 and maximum likelihood analysis of each catalytic domain of Tery_4685. Maximum likelihood phylogenetic tree (bootstraps $=100$ ) of individual btuR domains (domain only). Cyanobacteria are colored green. Bootstrap values $>50$ are noted. Parentheses indicate how many taxa within a collapsed clade. Scale bar represents average substitutions per site.

and respiration, iron flux (e.g., allocation) to CbiX relative to other biochemical iron sinks remains to be determined. However, Trichodesmium does contain the $\mathrm{B}_{12}$-dependent ribonucleotide reductase (NrdJ; Tery_0428) essential for the reduction of ribonucleotides to deoxyribonucleotides in DNA synthesis implicating a critical role for cobalamin in core metabolism.
Future studies examining both functional roles and enzyme efficiencies under co-limiting iron and cobalamin conditions are necessary to characterize these enzymes in the context of their ecophysiology.

Another line of evidence suggesting exogenous iron association to $\mathrm{B}_{12}$ biosynthesis is the fact that the Trichodesmium 
cobalt reductase orthologue to the Salmonella $\mathrm{B}_{12}$ flavodoxin ( $f l d A$; reciprocal best blast; evalue $<10^{-42}$ ), postulated to be responsible for the reduction of $\mathrm{Cob}(\mathrm{II})$ yrinic acid a,cdiamide to $\mathrm{Cob}(\mathrm{I})$ yrinic acid a,c-diamide (Figure 1; Fonseca and Escalante-Semerena, 2001), is the iron-stress flavodoxin gene isiB (Tery_1666) — which has been demonstrated to be directly regulated by iron bioavailability (Fonseca and EscalanteSemerena, 2001; Chappell and Webb, 2010; Walworth et al., 2016). It must be noted that Trichodesmium also contains another fldA that is not regulated by iron (Chappell and Webb, 2010), which could also possibly be involved in this reduction. Indeed, Trichodesmium is limited by iron across expansive ocean biomes (Sohm et al., 2011; Chappell et al., 2012; Hutchins and Boyd, 2016), which consequently limits new nitrogen inputs for primary production as a whole. Furthermore, since homologs of $i s i B$ are highly conserved in microbes possessing the anaerobic cobalamin biosynthesis pathway (Helliwell et al., 2016), these data suggest that $B_{12}$ biosynthesis and thus overall environmental bioavailability could be influenced by limiting iron. Interestingly, the BioCyc database (https://biocyc.org) implicates another gene, Tery_4461, as the cobalt reductase while the KEGG database (http://www. genome.jp/kegg/pathway.html) implicates isiB (Tery_1666) as above. However, it is compelling that $i s i B$ is indeed the true ortholog to the empirically investigated Salmonella $\mathrm{B}_{12}$ fldA (see above), which opens the door for future research in distinguishing the roles of these enzymes relative to cobalamin metabolism. As previously noted (Gaudu and Weiss, 2000), mutations to $f l d A$ homologs in genetically tractable organisms (e.g., E. coli) have been lethal, thereby prohibiting a traditional genetic approach to determine whether $f l d A$ is redundant to co(II)rrinoid reduction for adenosylation or whether it is solely responsible. Nonetheless, these data and other previous studies (Gaudu and Weiss, 2000; Heal et al., 2016) implicate a connective role between two essential micronutrients via prokaryotic core metabolism, and suggest that iron availability could directly influence not only nitrogen fixation, but also $\mathrm{B}_{12}$ biosynthesis in Trichodesmium.

\section{CONCLUSIONS}

We provide multiple lines of evidence both in culture and in situ that suggest Trichodesmium can transcribe and translate the full genomic pathway to biosynthesize and/or metabolize cobalamins. Our proof-of-concept meta-analyses from independent sources and analytical techniques open the door for more in-depth research into Trichodesmium B-vitamin dynamics. Since the synthesis of pseudo- $\mathrm{B}_{12}$ is an oxygenindependent pathway, Trichodesmium may have originally relied on pseudo- $\mathrm{B}_{12}$ biosynthesis in conjunction with early nitrogen fixation (another oxygen independent process). Hence, the $b l u B$ gene may have been acquired horizontally much later as evidenced by its clustering within Proteobacteria, although it is prohibitively difficult to confirm this from phylogeny alone. Future studies will be needed to specifically assess cobalamin vs pseudo- $\mathrm{B}_{12}$ production in Trichodesmium. Future experiments could include investigating isotopically labeled cobalamin- and iron- (co)-limiting molecular physiology with a range of biogeographically distinct isolates, in addition to analyzing B-vitamin dynamics directly from natural populations. Since large areas of the ocean are depleted in both $\mathrm{B}_{12}$ and bioavailable nitrogen, these data and other future experiments may highlight an additional keystone role that Trichodesmium populations could serve in oceanic biomes. Importantly, this role would distinguish Trichodesmium from other sympatric cyanobacteria that solely produce pseudo- $\mathrm{B}_{12}$, including other nitrogen fixers as mentioned above. Hence, cobalamin production may have aided in the ecological success of Trichodesmium if cobalamins are indeed used as a "currency" in exchange for other growth factors supplied by its associated epibionts, as potentially suggested by $\mathrm{B}_{12}$ auxotrophy in IMS101-associated epibiotic heterotrophs (Lee et al., 2017a; Romine et al., 2017). Further research with Trichodesmium isolates devoid of cyanobacterial epibionts is needed to investigate whether Trichodesmium can simultaneously produce both cobalamin and pseudo- $\mathrm{B}_{12}$, thereby potentially giving them the flexibility to exploit different $\mathrm{B}_{12}$ ecological niches in the oceans. Moreover, Trichodesmium and other cyanobacteria possess three copies of the btuR gene, predicted to be involved in de novo synthesis and corrinoid scavenging. Two of these three copies appear to be unique to cyanobacteria. Further characterization of these gene copies may provide critical insight into cyanobacterial $\mathrm{B}_{12}$ ecophysiology relative to other $\mathrm{B}_{12}$-producing microbes. Finally, cbiX (Tery_4741) and isiB (Tery_1666) genes within the cobalamin biosynthetic pathway may both be subject to influence by iron, thereby highlighting a potentially unrecognized role for iron limitation in simultaneously impacting Trichodesmium photosynthesis, nitrogen fixation, and $B_{12}$ biosynthesis. Experiments examining Trichodesmium iron limitation in the context of cobalamin limitation could reveal allocation dynamics of intracellular iron among critical iron-requiring metabolic pathways, and may indicate if the availability of this trace metal could potentially limit $\mathrm{B}_{12}$ synthesis in situ. Any preferential iron allocation strategies that may be employed by Trichodesmium under these conditions are currently unknown, but are certainly critical to understanding $B_{12}$ oceanic cycling if this diazotrophic cyanobacterium is indeed also a major euphotic zone source for cobalamins in the global oceans.

\section{AVAILABILITY OF DATA AND MATERIALS}

Raw reads have been deposited in NCBI's Gene Expression Omnibus (Edgar et al., 2002) and are accessible through GEO Series accession number GSE94951 (https://www.ncbi.nlm. nih.gov/geo/query/acc.cgi?acc=GSE94951). The raw read files used in this study have accession numbers GSM2492342 and GSM2492343. Experimental data are also available through the Biological and Chemical Oceanography Data Management Office (www.bco-dmo.org/project/551230 and www.bco-dmo.org/project/724451).

Protein spectral counts were downloaded directly from a previously published proteome study using these same IMS101 cell lines (Walworth et al., 2016). 


\section{SUBJECT CATEGORIES}

Integrated genomics and post-genomics approaches in microbial ecology.

\section{AUTHOR CONTRIBUTIONS}

DH, NW, ML, CS, PQ, F-XF, MS, EW, and SS-W: helped design and carry out the research; NW, DH, ML, MS, EW, and SS-W: were involved in writing and editing the paper.

\section{REFERENCES}

Altschul, S. F., Gish, W., Miller, W., Myers, E. W., and Lipman, D. J. (1990). Basic local alignment search tool. J. Mol. Biol. 215, 403-410. doi: 10.1016/S0022-2836(05)80360-2

Arrigo, K. R. (2005). Marine microorganisms and global nutrient cycles. Nature 437, 349-355. doi: 10.1038/nature04159

Banerjee, R., and Ragsdale, S. W. (2003). The many faces of vitamin B12: catalysis by cobalamin-dependent Enzymes1. Annu. Rev. Biochem. 72, 209-247. doi: 10.1146/annurev.biochem.72.121801.161828

Bergman, B., Sandh, G., Lin, S., Larsson, J., and Carpenter, E. J. (2013). Trichodesmium- a widespread marine cyanobacterium with unusual nitrogen fixation properties. FEMS Microbiol. Rev. 37, 286-302. doi: 10.1111/j.1574-6976.2012.00352.x

Bertrand, E. M., McCrow, J. P., Moustafa, A., Zheng, H., McQuaid, J. B., Delmont, T. O., et al. (2015). Phytoplankton-bacterial interactions mediate micronutrient colimitation at the coastal Antarctic sea ice edge. Proc. Natl. Acad. Sci. U.S.A. 112, 9938-9943. doi: 10.1073/pnas.1501615112

Bertrand, E. M., Moran, D. M., Mcllvin, M. R., Hoffman, J. M., Allen, A. E., and Saito, M. A. (2013). Methionine synthase interreplacement in diatom cultures and communities: implications for the persistence of B12 use by eukaryotic phytoplankton. Limnol. Oceanogr. 58, 1431-1450. doi: 10.4319/lo.2013.58.4.1431

Bolger, A. M., Lohse, M., and Usadel, B. (2014). Trimmomatic: a flexible trimmer for Illumina sequence data. Bioinformatics 30, 2114-2120. doi: 10.1093/bioinformatics/btu170

Bonnet, S., Webb, E. A., Panzeca, C., Karl, D. M., Capone, D. G., and Sanudo-Wilhelmy, S. (2010). Vitamin B12 excretion by cultures of the marine cyanobacteria Crocosphaera and Synechococcus. Limnol. Oceanogr. 55, 1959-1964. doi: 10.4319/lo.2010.55.5.1959

Brindley, A. A., Raux, E., Leech, H. K., Schubert, H. L., and Warren, M. J. (2003). A story of chelatase evolution. J. Biol. Chem. 278, 22388-22395. doi: $10.1074 /$ jbc.M302468200

Capella-Gutiérrez, S., Silla-Martínez, J. M., and Gabaldón, T. (2009). trimAl: a tool for automated alignment trimming in large-scale phylogenetic analyses. Bioinformatics 25, 1972-1973. doi: 10.1093/bioinformatics/btp348

Capone, D. G., Ferrier, M. D., and Carpenter, E. J. (1994). Amino acid cycling in colonies of the planktonic marine Cyanobacterium Trichodesmium thiebautii. Appl. Environ. Microbiol. 60, 3989-3995.

Capone, D. G., Zehr, J. P., Paerl, H. W., Bergman, B., and Carpenter, E. J. (1997). Trichodesmium, a globally significant marine Cyanobacterium. Science 276, 1221-1229. doi: 10.1126/science.276.5316.1221

Carlucci, A. F., Silbernagel, S. B., and McNally, P. M. (1969). Influence of temperature and solar radiation on persistence of vitamin B12, thiamine, and biotin in seawater. J. Phycol. 5, 302-305. doi: 10.1111/j.1529-8817.1969.tb02618.x

Chappell, P. D., and Webb, E. A. (2010). A molecular assessment of the iron stress response in the two phylogenetic clades of Trichodesmium. Environ. Microbiol. 12, 13-27. doi: 10.1111/j.1462-2920.2009.02026.x

Chappell, P. D., Moffett, J. W., Hynes, A. M., and Webb, E. A. (2012). Molecular evidence of iron limitation and availability in the global diazotroph Trichodesmium. ISME J. 6, 1728-1739. doi: 10.1038/ismej. 2012.13

\section{ACKNOWLEDGMENTS}

This work was funded by NSF research grants OCE-1260233, OCE-1260490, OCE-1657757, and OCE-143566.

\section{SUPPLEMENTARY MATERIAL}

The Supplementary Material for this article can be found online at: https://www.frontiersin.org/articles/10.3389/fmicb. 2018.00189/full\#supplementary-material

Croft, M. T., Lawrence, A. D., Raux-Deery, E., Warren, M. J., and Smith, A G. (2005). Algae acquire vitamin B12 through a symbiotic relationship with bacteria. Nature 438, 90-93. doi: 10.1038/nature04056

Croft, M. T., Warren, M. J., and Smith, A. G. (2006). Algae need their vitamins. Eukaryot. Cell 5, 1175-1183. doi: 10.1128/EC.00097-06

Doxey, A. C., Kurtz, D. A., Lynch, M. D., Sauder, L. A., and Neufeld, J. D. (2015). Aquatic metagenomes implicate Thaumarchaeota in global cobalamin production. ISME J. 9, 461-471. doi: 10.1038/ismej.2014.142

Droop, M. R. (2007). Vitamins, phytoplankton and bacteria: symbiosis or scavenging? J. Plankton Res. 29, 107-113. doi: 10.1093/plankt/fbm009

Edgar, R. C. (2004). MUSCLE: multiple sequence alignment with high accuracy and high throughput. Nucleic Acids Res. 32, 1792-1797. doi: $10.1093 /$ nar/gkh340

Edgar, R. C. (2010). Search and clustering orders of magnitude faster than BLAST. Bioinformatics 26, 2460-2461. doi: 10.1093/bioinformatics/btq461

Edgar, R., Domrachev, M., and Lash, A. E. (2002). Gene expression omnibus: NCBI gene expression and hybridization array data repository. Nucleic Acids Res. 30, 207-210. doi: 10.1093/nar/30.1.207

Escalante-Semerena, J. C. (2007). Conversion of cobinamide into adenosylcobamide in bacteria and archaea. J. Bacteriol. 189, 4555-4560. doi: 10.1128/JB.00503-07

Eschenmoser, A., and Wintner, C. E. (1977). Natural product synthesis and vitamin B12. Science 196, 1410-1420. doi: 10.1126/science.867037

Falkowski, P. G., Barber, R. T., and Smetacek, V. (1998). Biogeochemical controls and feedbacks on ocean primary production. Science 281, 200-207. doi: $10.1126 /$ science.281.5374.200

Fang, H., Kang, J., and Zhang, D. (2017). Microbial production of vitamin $\mathrm{B}_{12}$ : a review and future perspectives. Microb. Cell Fact. 16:15. doi: 10.1186/s12934-017-0631-y

Field, C. B., Behrenfeld, M. J., Randerson, J. T., and Falkowski, P. (1998). Primary production of the biosphere: integrating terrestrial and oceanic components. Science 281, 237-240. doi: 10.1126/science.281.5374.237

Fonseca, M. V., and Escalante-Semerena, J. C. (2001). An in vitro reducing system for the enzymic conversion of cobalamin to adenosylcobalamin. J. Biol. Chem. 276, 32101-32108. doi: 10.1074/jbc.M102510200

Gaudu, P., and Weiss, B. (2000). Flavodoxin mutants of Escherichia coli K-12. J. Bacteriol. 182, 1788-1793. doi: 10.1128/JB.182.7.1788-1793.2000

Giovannoni, S. J., Tripp, H. J., Givan, S., Podar, M., Vergin, K. L., Baptista, D., et al. (2005). Genome streamlining in a cosmopolitan oceanic bacterium. Science 309, 1242-1245. doi: 10.1126/science.1114057

Hazra, A. B., Han, A. W., Mehta, A. P., Mok, K. C., Osadchiy, V., Begley, T. P., et al. (2015). Anaerobic biosynthesis of the lower ligand of vitamin B12. Proc. Natl. Acad. Sci. U.S.A. 112, 10792-10797. doi: 10.1073/pnas.1509132112

Heal, K. R., Qin, W., Ribalet, F., Bertagnolli, A. D., Coyote-Maestas, W., Hmelo, L. R., et al. (2016). Two distinct pools of B12 analogs reveal community interdependencies in the ocean. Proc. Natl. Acad. Sci. U.S.A. 114, 364-369. doi: $10.1073 /$ pnas. 1608462114

Helliwell, K. E., Lawrence, A. D., Holzer, A., Kudahl, U. J., Sasso, S., Kräutler, B., et al. (2016). Cyanobacteria and eukaryotic algae use different chemical variants of Vitamin B12. Curr. Biol. 26, 999-1008. doi: 10.1016/j.cub.2016. 02.041

Hewson, I., Poretsky, R. S., Dyhrman, S. T., Zielinski, B., White, A. E., Tripp, H. J., et al. (2009). Microbial community gene expression within colonies of 
the diazotroph, Trichodesmium, from the Southwest Pacific Ocean. ISME J. 3, 1286-1300. doi: 10.1038/ismej.2009.75

Hutchins, D. A., and Boyd, P. W. (2016). Marine phytoplankton and the changing ocean iron cycle. Nat. Clim. Change 6, 1072-1079. doi: 10.1038/nclimate3147

Hutchins, D. A., Mulholland, M. R., and Fu, F.-X. (2009). Nutrient cycles and marine microbes in a $\mathrm{CO}_{2}$-enriched ocean. Oceanography 22, 128-145. doi: 10.5670/oceanog.2009.103

Hutchins, D. A., Walworth, N. G., Webb, E. A., Saito, M. A., Moran, D., McIlvin, M. R., et al. (2015). Irreversibly increased nitrogen fixation in Trichodesmium experimentally adapted to elevated carbon dioxide. Nat. Commun. 6:8155. doi: $10.1038 /$ ncomms9155

Knauer, G. A., Martin, J. H., and Gordon, R. M. (1982). Cobalt in north-east Pacific waters. Nature 297, 49-51. doi: 10.1038/297049a0

Langmead, B., and Salzberg, S. L. (2012). Fast gapped-read alignment with Bowtie 2. Nat. Methods 9, 357-359. doi: 10.1038/nmeth.1923

Lee, M. D., Walworth, N. G., McParland, E. L., Fu, F.-X., Mincer, T. J., Levine, N. M. et al. (2017a). The Trichodesmium consortium: conserved heterotrophic co-occurrence and genomic signatures of potential interactions. ISME J. 11, 1813-1824. doi: 10.1038/ismej.2017.49

Lee, M. D., Webb, E. A., Walworth, N. G., Fu, F.-X., Held, N. A., Saito, M. A., et al. (2017b). Transcriptional activities of the microbial consortium living with the marine nitrogen-fixing Cyanobacterium Trichodesmium reveal potential roles in community-level nitrogen cycling. Appl. Environ. Microbiol. 84:e02026-17-16. doi: 10.1128/AEM.02026-17

Leech, H. K., Raux, E., McLean, K. J., Munro, A. W., Robinson, N. J., Borrelly, G. P. M., et al. (2003). Characterization of the cobaltochelatase CbiXL: evidence for a $4 \mathrm{Fe}-4 \mathrm{~S}$ center housed within an MXCXXC motif. J. Biol. Chem. 278, 41900-41907. doi: 10.1074/jbc.M306112200

Lynch, M., and Marinov, G. K. (2015). The bioenergetic costs of a gene. Proc. Natl. Acad. Sci. U.S.A. 112, 15690-15695. doi: 10.1073/pnas.1514974112

Mehta, A. P., Abdelwahed, S. H., Fenwick, M. K., Hazra, A. B., Taga, M. E., Zhang, Y., et al. (2015). Anaerobic 5-Hydroxybenzimidazole formation from aminoimidazole ribotide: an unanticipated intersection of Thiamin and Vitamin B12 biosynthesis. J. Am. Chem. Soc. 137, 10444-10447. doi: 10.1021/jacs.5b03576

Mohn, W. W., and Tiedje, J. M. (1992). Microbial reductive dehalogenation. Microbiol. Rev. 56, 482-507.

Monteverde, D. R., Gómez-Consarnau, L., Suffridge, C., and Sañudo-Wilhelmy, S. A. (2016). Life's utilization of B vitamins on early Earth. Geobiology 15, 3-18. doi: $10.1111 / \mathrm{gbi} .12202$

Morrissey, J., and Bowler, C. (2012). Iron utilization in marine cyanobacteria and eukaryotic algae. Front. Microbiol. 3:43. doi: 10.3389/fmicb.2012. 00043

Nowack, E. C., Melkonian, M., and Glöckner, G. (2008). Chromatophore genome sequence of paulinella sheds light on acquisition of photosynthesis by Eukaryotes. Curr. Biol. 18, 410-418. doi: 10.1016/j.cub.2008.02.051

Panzeca, C., Beck, A. J., Leblanc, K., Taylor, G. T., Hutchins, D. A., and Sañudo-Wilhelmy, S. A. (2008). Potential cobalt limitation of vitamin $B_{12}$ synthesis in the North Atlantic Ocean. Global Biogeochem. Cycles 22:GB2029. doi: 10.1029/2007GB003124

Panzeca, C., Tovar-Sanchez, A., Agustí, S., Reche, I., Duarte, C. M., Taylor, G. T., et al. (2006). B vitamins as regulators of phytoplankton dynamics. EOS Trans. Am. Geophys. Union 87, 593-596. doi: 10.1029/2006EO520001

Rauhut, R., and Klug, G. (1999). mRNA degradation in bacteria. FEMS Microbiol Rev 23, 353-370. doi: 10.1111/j.1574-6976.1999.tb00404.x

Robinson, M. D., McCarthy, D. J., and Smyth, G. K. (2010). edgeR: a Bioconductor package for differential expression analysis of digital gene expression data. Bioinformatics 26, 139-140. doi: 10.1093/bioinformatics/btp616

Rodionov, D. A. (2003). Comparative genomics of the vitamin B12 metabolism and regulation in prokaryotes. J. Biol. Chem. 278, 41148-41159. doi: $10.1074 /$ jbc.M305837200

Romine, M. F., Rodionov, D. A., Maezato, Y., Anderson, L. N., Nandhikonda, P., Rodionova, I. A., et al. (2017). Elucidation of roles for vitamin B12 in regulation of folate, ubiquinone, and methionine metabolism. Proc. Natl. Acad. Sci. U.S.A. 114, E1205-E1214. doi: 10.1073/pnas.1612360114

Rouco, M., Haley, S. T., and Dyhrman, S. T. (2016). Microbial diversity within the Trichodesmium holobiont. Environ. Microbiol. 18, 5151-5160. doi: $10.1111 / 1462-2920.13513$
Saito, M. A., Rocap, G., and Moffett, J. W. (2005). Production of cobalt binding ligands in a Synechococcus feature at the Costa Rica upwelling dome. Limnol. Oceanogr. 50, 279-290. doi: 10.4319/lo.2005.50.1.0279

Santoro, A. E., Dupont, C. L., Richter, R. A., Craig, M. T., Carini, P., McIlvin, M. R., et al. (2015). Genomic and proteomic characterization of "Candidatus Nitrosopelagicus brevis": an ammonia-oxidizing archaeon from the open ocean. Proc. Natl. Acad. Sci. U.S.A. 112, 1173-1178. doi: $10.1073 /$ pnas. 1416223112

Sañudo-Wilhelmy, S. A., Gómez-Consarnau, L., Suffridge, C., and Webb, E. A. (2014). The role of B vitamins in marine biogeochemistry. Annu. Rev. Mar. Sci. 6, 339-367. doi: 10.1146/annurev-marine-120710-100912

Sohm, J. A., Webb, E. A., and Capone, D. G. (2011). Emerging patterns of marine nitrogen fixation. Nat. Rev. Microbiol. 9, 499-508. doi: 10.1038/nrmicro2594

Stadtman, E. R., Overath, P., Eggerer, H., and Lynen, F. (1960). The role of biotin and vitamin B12 coenzyme in propionate metabolism. Biochem. Biophys. Res. Commun. 2, 1-7. doi: 10.1016/0006-291X(60)90252-7

Stamatakis, A. (2015). Using RAxML to infer phylogenies. Curr. Protoc. Bioinformatics 51, 6.14.1-6.14.14. doi: 10.1002/0471250953. bi0614s51

Suffridge, C., Cutter, L., and Sa-udo-Wilhelmy, S. A. (2017). A new analytical method for direct measurement of particulate and dissolved B-vitamins and their congeners in seawater. Front. Mar. Sci. 4, 175. doi: $10.3389 /$ fmars.2017.00011

Sullivan, M. B., Krastins, B., Hughes, J. L., Kelly, L., Chase, M., Sarracino, D., et al. (2009). The genome and structural proteome of an ocean siphovirus: a new window into the cyanobacterial "mobilome." Environ. Microbiol. 11, 2935-2951. doi: 10.1111/j.1462-2920.2009.02081.x

Taga, M. E., and Walker, G. C. (2008). Pseudo-B12 joins the cofactor family. J. Bacteriol. 190, 1157-1159. doi: 10.1128/JB.01892-07

Tatusova, T., Ciufo, S., Federhen, S., Fedorov, B., McVeigh, R., O’Neill, K., et al. (2015). Update on RefSeq microbial genomes resources. Nucleic Acids Res. 43, D599-D605. doi: 10.1093/nar/gku1062

Walworth, N. G., Fu, F.-X., Webb, E. A., Saito, M. A., Moran, D., Mcllvin, M. R., et al. (2016). Mechanisms of increased Trichodesmium fitness under iron and phosphorus co-limitation in the present and future ocean. Nat. Commun. 7:12081. doi: $10.1038 /$ ncomms 12081

Walworth, N., Pfreundt, U., Nelson, W. C., Mincer, T., Heidelberg, J. F., Fu, F., et al. (2015). Trichodesmium genome maintains abundant, widespread noncoding DNA in situ, despite oligotrophic lifestyle. Proc. Natl. Acad. Sci. U.S.A. 112, 4251-4256. doi: 10.1073/pnas.1422332112

Warren, M. J., Raux, E., Schubert, H. L., and Escalante-Semerena, J. C. (2002). The biosynthesis of adenosylcobalamin (vitamin B12). Nat. Prod. Rep. 19, 390-412. doi: $10.1039 / \mathrm{b} 108967 \mathrm{f}$

Wienhausen, G., Noriega-Ortega, B. E., Niggemann, J., Dittmar, T., and Simon, M. (2017). The exometabolome of two model strains of the roseobacter group: a marketplace of microbial metabolites. Front. Microbiol. 8:1985. doi: $10.3389 /$ fmicb.2017.01985

Woodson, J. D., and Escalante-Semerena, J. C. (2003). CbiZ, an amidohydrolase enzyme required for salvaging the coenzyme B. Proc. Natl. Acad. Sci. U.S.A. 101, 3591-3596. doi: 10.1073/pnas.0305939101

Yan, J., Bi, M., Bourdon, A. K., Farmer, A. T., Wang, P.-H., Molenda, O., et al. (2018). Purinyl-cobamide is a native prosthetic group of reductive dehalogenases. Nat. Chem. Biol. 14, 8-14. doi: 10.1038/ nchembio. 2512

Zehr, J. P. (2011). Nitrogen fixation by marine cyanobacteria. Trends Microbiol. 19, 162-173. doi: 10.1016/j.tim.2010.12.004

Conflict of Interest Statement: The authors declare that the research was conducted in the absence of any commercial or financial relationships that could be construed as a potential conflict of interest.

Copyright (๑) 2018 Walworth, Lee, Suffridge, Qu, Fu, Saito, Webb, Sañudo-Wilhelmy and Hutchins. This is an open-access article distributed under the terms of the Creative Commons Attribution License (CC BY). The use, distribution or reproduction in other forums is permitted, provided the original author(s) and the copyright owner are credited and that the original publication in this journal is cited, in accordance with accepted academic practice. No use, distribution or reproduction is permitted which does not comply with these terms. 